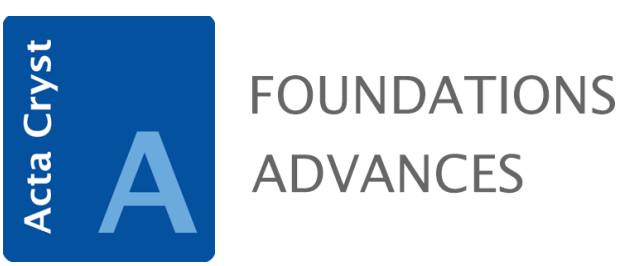

Volume 74 (2018)

Supporting information for article:

A tenfold twin of the CrB structure type

Wolfgang Hornfeck 


\author{
Supporting information for the article \\ On Tenfold Twins of the CrB Structure Type \\ authored by \\ Wolfgang Hornfeck \\ Institute of Physics of the Czech Academy of Sciences \\ Department of Structure Analysis \\ Na Slovance 2, 18221 Praha 8, Czech Republic
}

August 20, 2018

\title{
Contents
}

1 Crystallographic annotations $\quad 2$

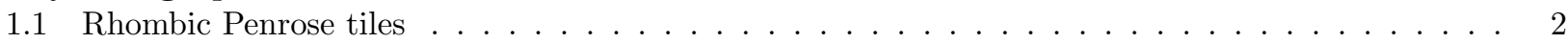

1.2 Penrose tiles in the tenfold twin structure $($ case $\kappa=1) \ldots \ldots \ldots$

2 Mathematical derivations $\mathbf{5}$

2.1 Counting the number of 1 's in the sequence $0,0,0,1,0,0,1,0,0,1,0,1, \ldots \ldots \ldots$

2.2 Equivalence of certain expressions involving floor functions . . . . . . . . . . . . . . 7

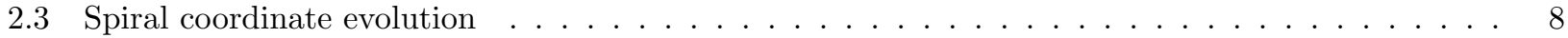

2.3.1 'Higher-dimensional' embedding $(2 \mathrm{D}$ into $3 \mathrm{D}) \ldots \ldots \ldots$

2.3 .2 Multiplicities $\mu_{k, \ell}^{(n)} \ldots \ldots \ldots \ldots \ldots \ldots$

2.3.3 Spiral coordinate evolution .......................... . . 10

3 Computer code $\quad 11$

3.1 Mathematica script for the $2 \mathrm{D}$ case of the tenfold twin structure $(\kappa=1$ case $) \ldots . . . . .11$

3.2 Mathematica script for the $3 \mathrm{D}$ case of the tenfold twin structure $(\kappa=1$ case $)$. . . . . . . . 12 


\section{Crystallographic annotations}

\subsection{Rhombic Penrose tiles}

The rhombic Penrose tiling consists of two rhombs, a thin one and a thick one, which angles are multiples of $\alpha=\pi / 5=36^{\circ}$. In particular, the thin rhomb has an acute (subscript $a$ ) angle of $\alpha\left(36^{\circ}\right)$ and an obtuse (subscript $o$ ) angle of $4 \alpha\left(144^{\circ}\right)$, while the thick rhomb has an acute angle of $2 \alpha\left(72^{\circ}\right)$ and an obtuse one of $3 \alpha\left(108^{\circ}\right)$. Their four diagonals, together with the edge length, here set to unity, define a set of five distinct 'minimal' lengths occurring in such a tiling (Fig. S1).

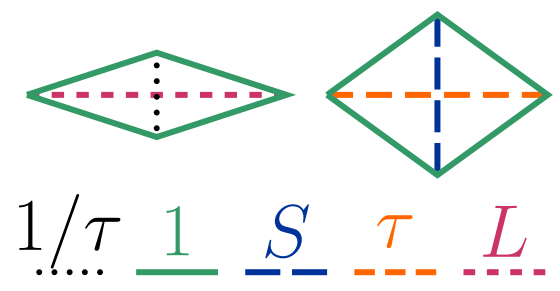

Fig. S1: Five distances in the thin and thick rhombic Penrose tiles.

In a quasiperiodic Penrose tiling, following the quasiperiodicity-enforcing matching rules indicated by the colored circular markings on the tiles, only eight distinct vertex configurations are allowed (Fig. S2).

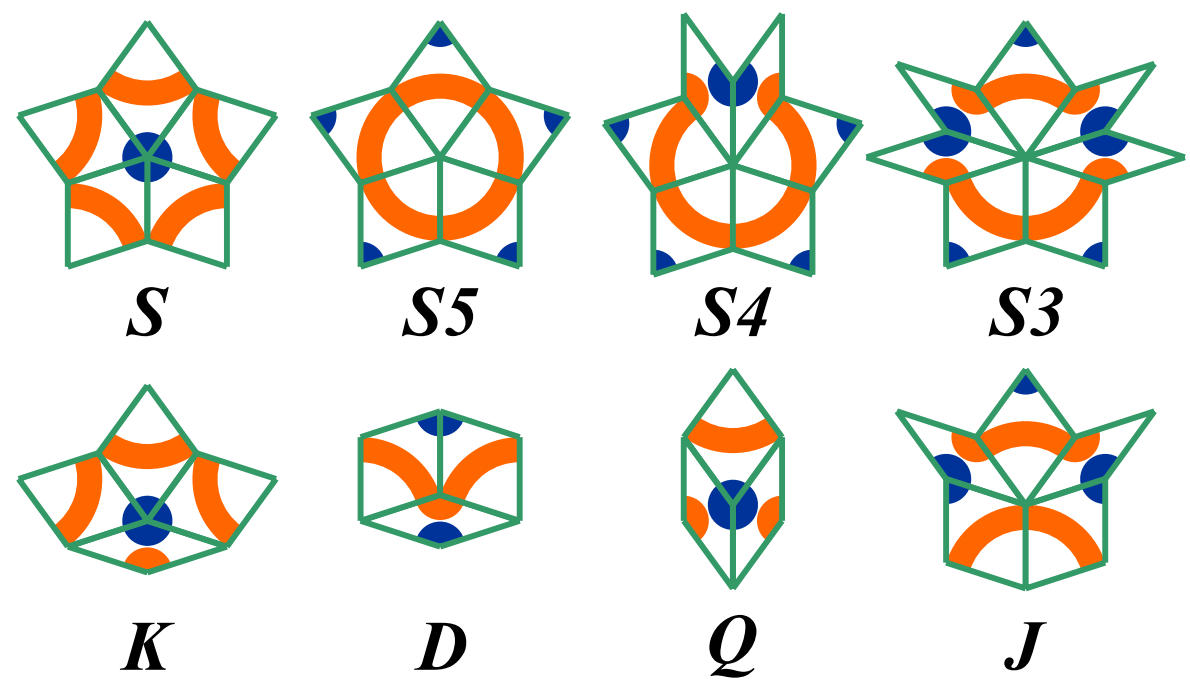

Fig. S2: Eight possible vertex configurations of the rhombic Penrose tiling with their designations due to de Bruijn (N. G. de Bruijn, (1981). Algebraic theory of Penrose's non-periodic tilings of the plane. I, II. Kon. Nederl. Akad. Wetensch. Proc. Ser. A84 (= Indag. Math.), 39-66).

\subsection{Penrose tiles in the tenfold twin structure (case $\kappa=1$ )}

The tenfold twin structure can be described on the basis of a tiling composed of thin and thick Penrose rhombs, but it is obvious from the start that this can only be true on the level of the undecorated tiles, since the regions within the twin domains are composed of finite patches of bulk NiZr, i.e. cut-outs of a periodic crystal structure, and thus a periodic arrangement of Penrose rhombs. For a quasiperiodic arrangement of Penrose rhombs the decorated tiles have to fulfill the matching rules according to their inscribed markings (colored circular/annular sectors). Of all the possible vertex configurations, there are only eight ones fulfilling this condition (Fig. S2). From these, only the vertex configurations of type $K$ and $Q$ are found in the tenfold twin tiling. Yet even the occurrence of these vertex decorations is not possible for all vertices, if one considers tiles decorated according 
to the matching rules. For instance, the vertex configurations $K$ and $Q$ are forming an overlapping pair at the long boundary of a CrB type unit cell, such that the shared tile cannot fulfill the matching rules for both vertex configurations simultaneously (Fig. S3). Since the flipping of a tile contributes to the phasonic disorder of quasicrystals and the shared tile experiences a dilemma regarding its orientation (geometric frustration), one could call this conflicting state of tile arrangements a phasonic frustration.
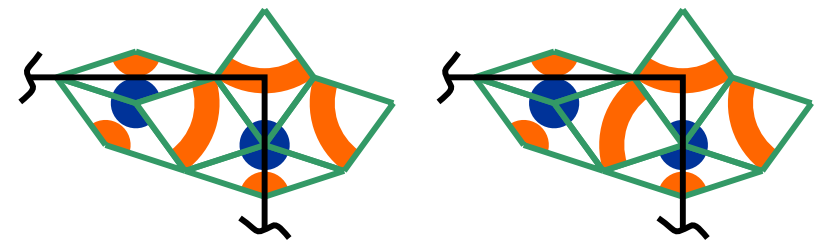

Fig. S3: Overlapping pair of vertex configurations $Q$ and $K$, with their shared tile experiencing phasonic frustration, matching either such as to complete the $K$ configuration (left) or the $Q$ one (right).

In a similar fashion the vertex configuration $Q$ forms an overlapping pair with itself at the short boundary of a CrB type unit cell, and again, the shared tile cannot fulfill the matching rules simultaneously. Moreover, the restrictions imposed by translation periodicity does not allow for a second $Q$ configuration at all, i.e. if the decoration of the tiles, indicating the matching rules, is expected to follow this symmetry (Fig. S4). In both cases the decoration of the tiles also breaks the mirror symmetry of an undecorated tiling, a conflict which cannot be resolved by any arrangement of tiles whatsoever.
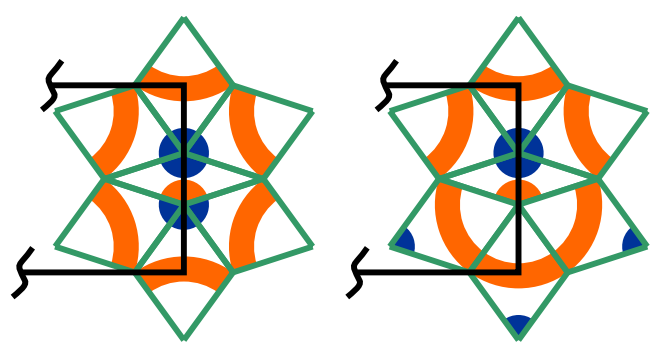

Fig. S4: Overlapping pair of vertex configuration $Q$ with itself, with their shared tile experiencing phasonic frustration (left, only one state is shown, which represents a matching $K$ configuration in the upper part).

Thus, one has the case of a compatibility of the undecorated tiles, while there exists a non-compatibility of the decorated ones. In addition to this non-compatibility of tiles, the tiling associated with the tenfold twin contains three additional vertex configurations, other than the eight vertex configurations shown in Fig. S2.
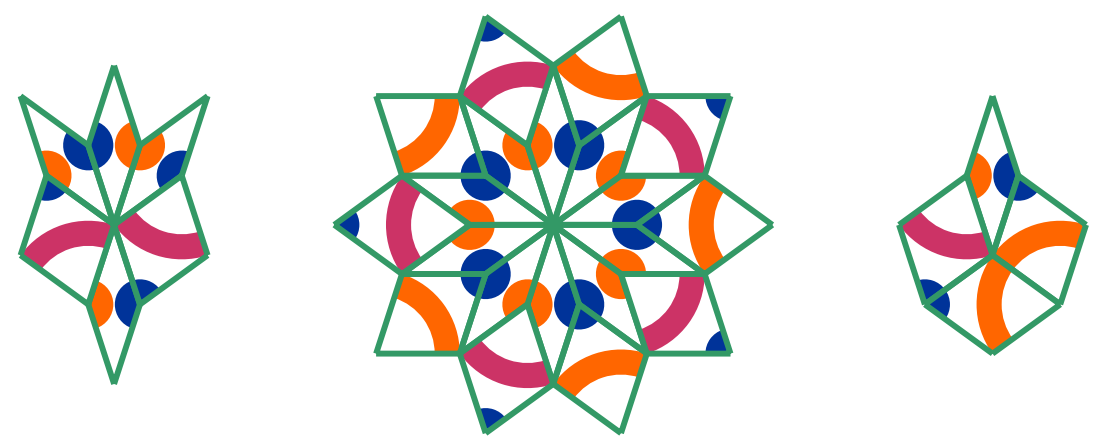

Fig. S5: Vertex configurations present in the tenfold twin structure showing the mismatch of the circular/annular decorations on the Penrose tiles. 


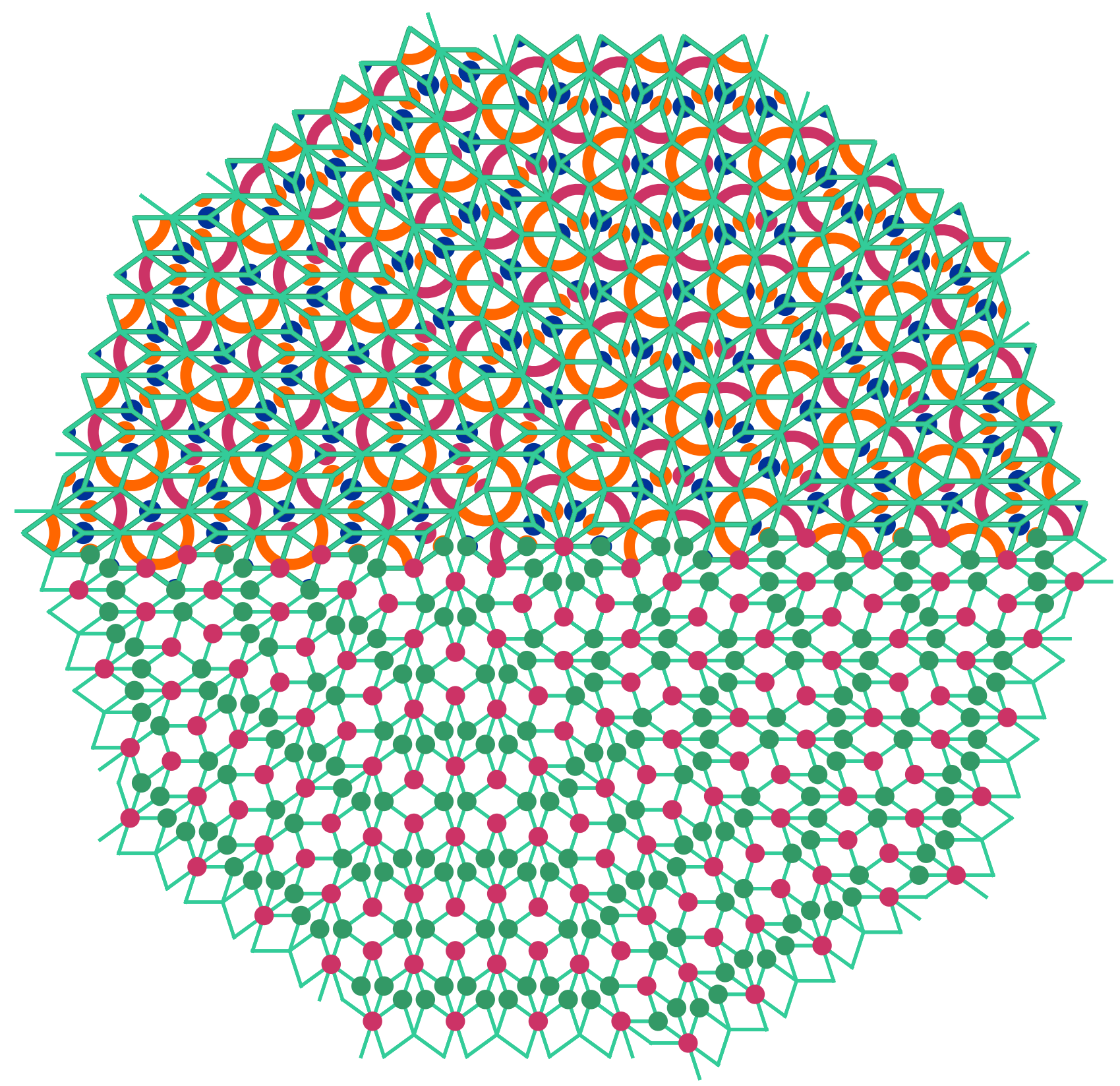

Fig. S6: Central patch of a cyclic intergrowth of ten domains $(\kappa=1$ case, shift $\sigma=\sqrt{5} / 2)$ with decorated tiles (upper half) and decorated vertices (lower half; red and green nodes represent vertex configurations forbidden and allowed in a quasiperiodic rhombic Penrose tiling, respectively). 


\section{Mathematical derivations}

\subsection{Counting the number of 1 's in the sequence $0,0,0,1,0,0,1,0,0,1,0,1, \ldots$}

In the following we shortly derive a formula counting the number of 1 's in the string up to a given limit $P$.

For this purpose we use the hierarchy of the string on the first level. Then $P$ is either coinciding with a site of the type $2 n^{2}$ or located between two sites $2 n^{2}$ and $2(n+1)^{2}$. In both cases it is important to know the number of 1's up to $2 n^{2}$, which already is the answer for the first case. We observe that there are zero 1 's up to the number two $(n=1)$, two 1's up to the number eight $(n=2)$, six 1's up to the number $18(n=3)$, and eventually

$$
\sum_{i=0}^{n-1} 2 i=n^{2}-n
$$

1's up to the number $2 n^{2}$. Now, if $P$ does not coincide with a site of the type $2 n^{2}$ there may be additional 1 's to count, depending on the exact position of $P$ between two sites $2 n^{2}$ and $2(n+1)^{2}$. In general, there will be

$$
2(n+1)^{2}-2 n^{2}=4 n+2
$$

sites in this interval, of which (cf. equation 1)

$$
(n+1)^{2}-(n+1)-\left(n^{2}-n\right)=2 n
$$

will be 1's, as can be seen from Fig. 11 counting the black boxes (and, of course, $2 n+2$ will be 0 's, due to complementarity of the string). The first site will always be a 0 with 1 's and 0 's alternating afterwards until the middle of the interval, where an additional 0 shifts the alternating pattern of 1 's and 0 's one step further apart. It is especially this shift which has to be accounted for. Regarding the first half of the interval a 1 is to be counted at every second step. This is achieved by applying the floor function in the form of $\lfloor\cdot / 2\rfloor$. The appropriate argument for this partial counting function, in order to let it start from zero, is given as $P-2 n^{2}$, thus obtaining $\left\lfloor\left(P-2 n^{2}\right) / 2\right\rfloor$. In order to account for the offset of one in the second half of the interval one has to adjust this to $\left\lfloor\left(P-2 n^{2}-1\right) / 2\right\rfloor$. To avoid a case distinction, and obtain a single, comprehensive formula, the floor function is invoked another time, now in order to assign 0 or 1 , depending on whether $P$ is located in the first or second half of the interval, respectively. This is accomplished by $\left\lfloor\left(P-2 n^{2}\right) /(2 n+1)\right\rfloor$, in which the denominator denotes half of the sites in the interval (cf. equation 2). Combining the results yields

$$
\begin{aligned}
\#_{1}(S) & =n^{2}-n+\left\lfloor\frac{1}{2}\left(P-2 n^{2}-\left\lfloor\frac{P-2 n^{2}}{2 n+1}\right\rfloor\right)\right\rfloor \\
& =\left\lfloor n^{2}-n+\frac{1}{2}\left(P-2 n^{2}-\left\lfloor\frac{P-2 n^{2}}{2 n+1}\right\rfloor\right)\right\rfloor \\
& =\left\lfloor\frac{1}{2}\left(2 n^{2}-2 n+P-2 n^{2}-\left\lfloor\frac{P-2 n^{2}}{2 n+1}\right\rfloor\right)\right\rfloor \\
& =\left\lfloor\frac { 1 } { 2 } \left( P-2 n+\left\lfloor-\frac{P-2 n^{2}}{2 n+1} \mid\right) \mid\right.\right. \\
& =\left\lfloor\frac{1}{2}\left[P-2 n-\frac{P-2 n^{2}}{2 n+1}\right\rceil \mid\right. \\
& =\left\lfloor\frac{1}{2}\left[\frac{2 n P+P-4 n^{2}-2 n-P+2 n^{2}}{2 n+1}\right\rceil\right] \\
& =\left\lfloor\frac{1}{2}\left\lceil 2 \frac{n(P-n-1)}{2 n+1}\right\rceil\right. \\
& =\left\lceil\frac{n(P-n-1)}{2 n+1}\right\rceil \\
& =\left\lceil\frac{2 n P-2 n^{2}-2 n-(2 n+1)}{2(2 n+1)}\right\rceil \\
& =\left\lceil\frac{n(P-n-2)-1 / 2}{2 n+1}\right\rceil
\end{aligned}
$$


for a function counting the number of 1's in the binary string $S$ up to a given limit $P$. The simplifications are due to the inclusion of integers into the floor functions (steps 5 and 8) and by using the identities $-\lfloor x\rfloor=\lceil-x\rceil$ (step 7) and $\lfloor(1 / 2)\lceil 2 x\rceil\rfloor=\lceil x-1 / 2\rceil$ (step 11), respectively. Note, that $n=\lfloor\sqrt{P / 2}\rfloor$, following from $2 n^{2}$ being the lower boundary of the interval. Eventually, since the alternating pattern of 0's and 1's becomes ever more dominant, its part in the string increases monotonically in length for increasing $P$, the ratio $\#_{S}(1) / P$ approaches $1 / 2$ asymptotically from below for $P \rightarrow \infty$. 


\subsection{Equivalence of certain expressions involving floor functions}

In the following we proof the equivalence of the expression

$$
\lfloor\sqrt{n-1}\rfloor=\lfloor(n-1) / \nu\rfloor,
$$

where $\nu=\lfloor\sqrt{n}+1 / 2\rfloor$. Plotting the argument of the outer floor functions of both the left and right hand side of the equation separately (Fig. S7) one observes that both functions, one continuous, the other one discontinuous, intersect each other for all values of $n=m^{2}+1$ with $m$ being a positive integer.

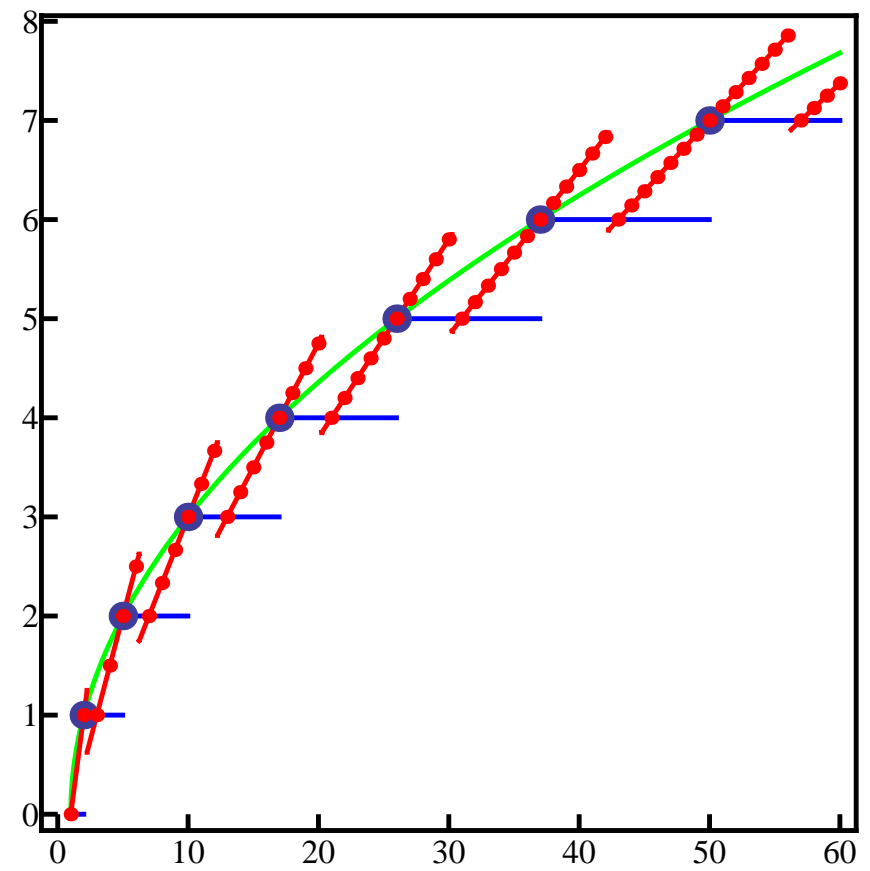

Fig. S7: On the equivalence of $\lfloor\sqrt{n-1}\rfloor=\lfloor(n-1) / \nu\rfloor$. Shown are the plots for $(n-1) / \nu$ (red line segments and red discs for integer $n$ ), $\sqrt{n-1}$ (green curve), and $\lfloor\sqrt{n-1}\rfloor$ (blue step function). If the floor operation is performed on $(n-1) / \nu$ the result will a projection of the (red disc) integers onto the (blue) step function. Larger blue discs, underlying the red ones, mark the integers $n=m^{2}+1$.

Plugging this in yields

$$
m=\left\lfloor m^{2} / \nu\right\rfloor
$$

In order for equation 15 to become true, $\nu=m$ must hold true, i.e.

$$
\left\lfloor\sqrt{m^{2}+1}+1 / 2\right\rfloor=\left\lfloor\sqrt{m^{2}+1}-m+1 / 2\right\rfloor+m \stackrel{!}{=} m
$$

This is true, if

$$
\left\lfloor\sqrt{m^{2}+1}-m+1 / 2\right\rfloor=0
$$

which is true, if $\sqrt{m^{2}+1}-m<1 / 2$ for all $m>0$. The function on the left hand side of this inequality is strictly monotonic decreasing, with its first derivative

$$
-1+\frac{m}{\sqrt{m^{2}+1}}<0
$$

for all $m>0$. In particular, the denominator $\sqrt{m^{2}+1}$ approximates the enumerator $m$ asymptotically from above, i.e.

$$
\lim _{m \rightarrow \infty}\left(\sqrt{m^{2}+1}-m\right)=0
$$

Since $\sqrt{m^{2}+1}>m$ the second term of the first derivative is always smaller than unity. Finally, since $\sqrt{m^{2}+1}-$ $m<1 / 2$ for all real $m>3 / 4$, hence it is true for all positive integers, thereby confirming equation 15 . 


\subsection{Spiral coordinate evolution}

\subsection{1 'Higher-dimensional' embedding (2D into 3D)}

Following the simplified treatment of embedding the spiral inclination pattern into a 2D triangular lattice we show here its (rather trivial) projection from a 3D primitive cubic lattice (Fig. S8 and S9)

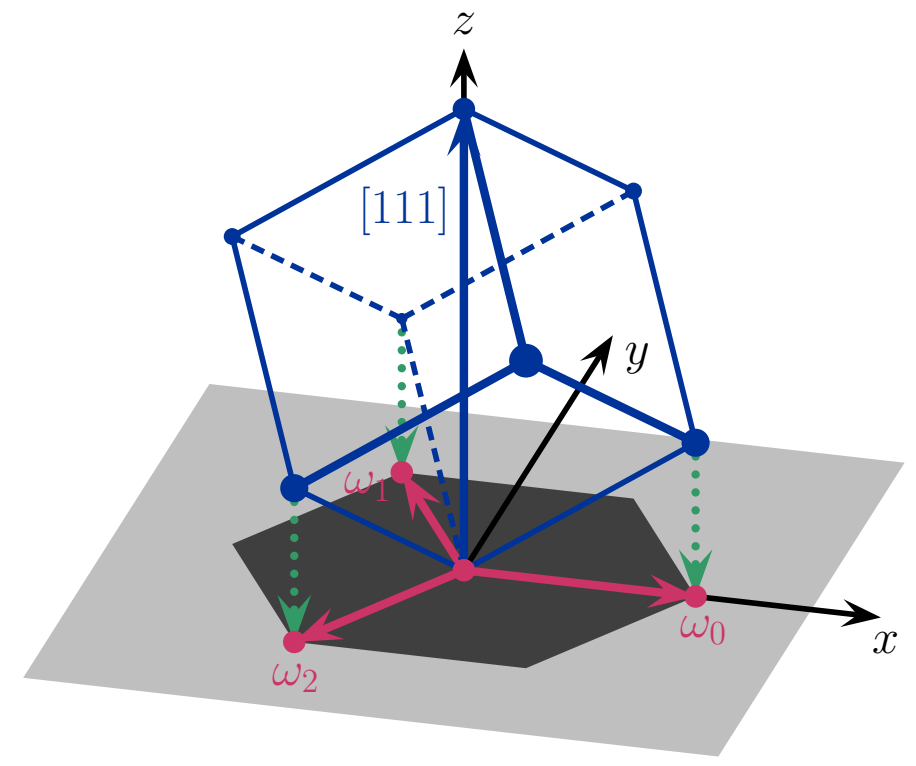

Fig. S8: Projection of a 3D cube with lattice parameter $a=\sqrt{3 / 2}$ ) (blue) along its [111] direction, to yield the basis vectors of a $2 \mathrm{D}$ triangular lattice (red), corresponding to the third roots of unity $\omega_{0}=(1,0), \omega_{1}=$ $(1 / 2)(-1, \sqrt{3})$, and $\omega_{2}=(1 / 2)(-1,-\sqrt{3})$ in the complex plane. Projecting a $5 \mathrm{D}$ cube along its [11111] direction yields the basis vectors of a $2 \mathrm{D}$ pentagonal $\mathbb{Z}$ module instead.

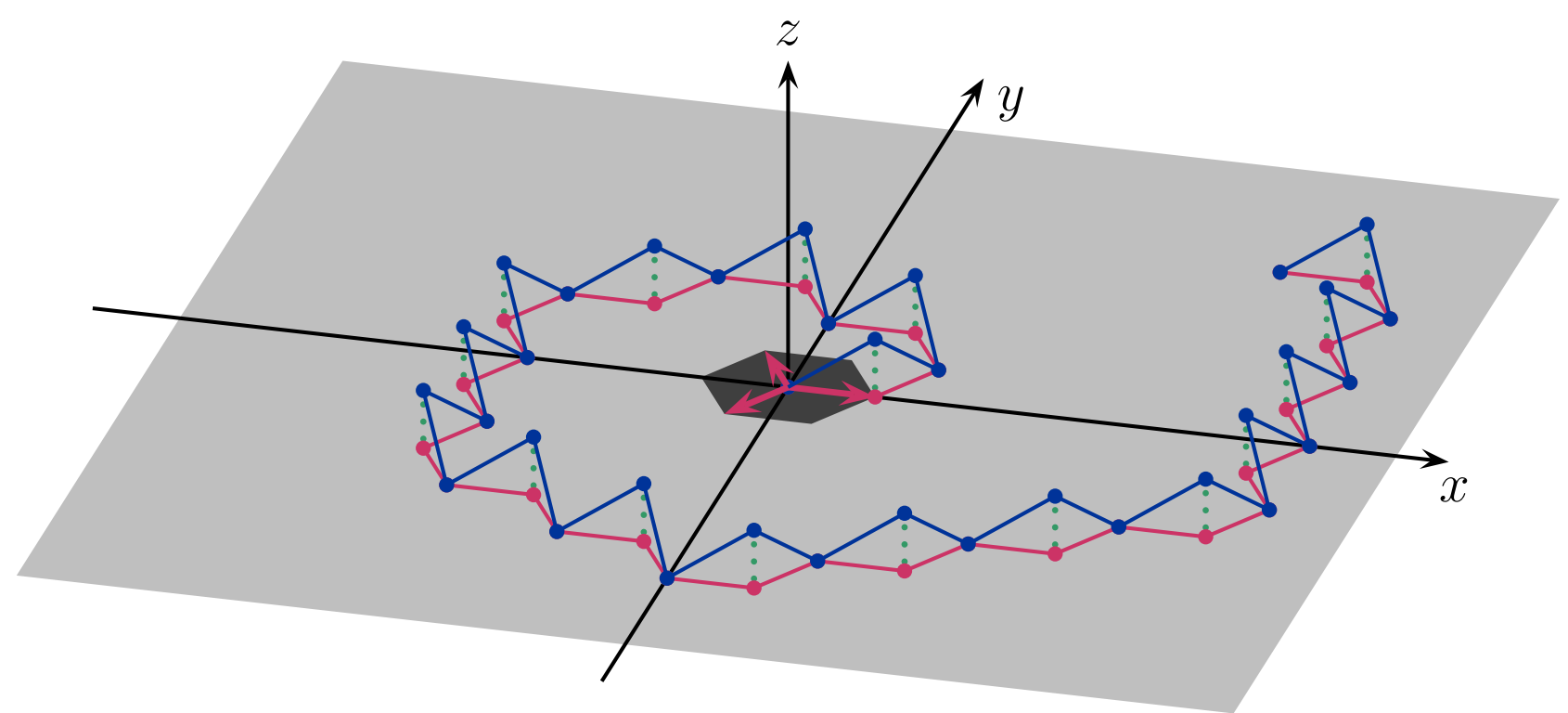

Fig. S9: Spiral path in the two-dimensional complex plane with the third roots of unity highlighted as basis vectors of a triangular lattice (red points, lines, and arrows) and the corresponding primitive cubic lattice path in three dimensions consisting of alternating up and down movements (blue points and lines). 


\subsubsection{Multiplicities $\mu_{k, \ell}^{(n)}$}

The following graph collects the multiplicity values $\mu_{k, \ell}^{(n)}$ according to their azimuthal and radial coordinates, $k$ and $\ell$, respectively.

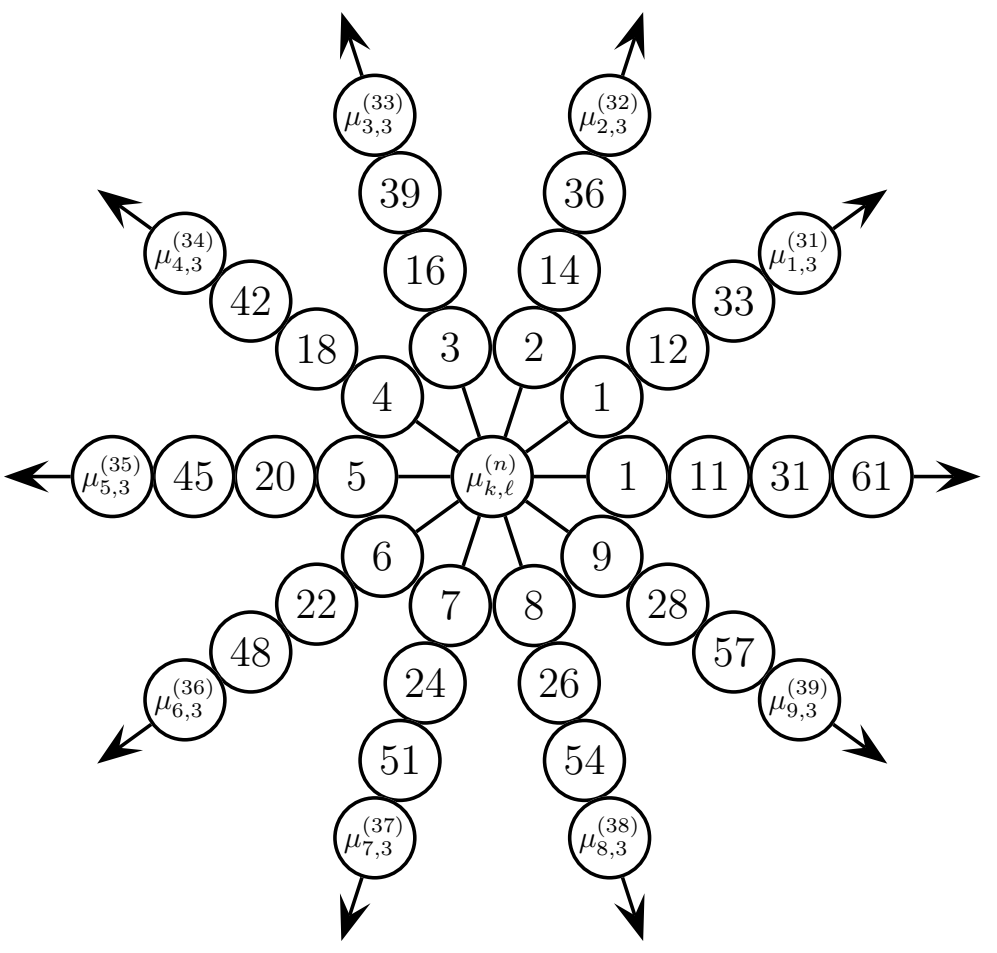

Fig. S10: Spiral multiplicities $\mu_{k, \ell}^{(n)}$ at which a change in in the alternation of directions occurs. 


\subsubsection{Spiral coordinate evolution}

The coordinate evolution can be best studied by writing down the basic vector multiplicity decuples and follow their consecutive changes Table S1.

Tab. S1: Basis vector multiplicity decuples, $\left[m_{0}, \cdots, m_{9}\right]$, and $\mathbb{Z}$ module quintuples, $\left[z_{0}, \cdots, z_{5}\right]$, for 50 consecutive points of a single spiral. The zeroth point is given by [0000000000] $=[00000]$. For reasons of simplicity and clarity, and also because only multiplicities/integer coordinates from 0 to 9 actually appear for the chosen points, commas are omitted. Negative values, appearing in the $\mathbb{Z}$ module quintuples, are overlined.

\begin{tabular}{|c|c|c|c|c|c|}
\hline$n$ & {$\left[m_{0}, \cdots, m_{9}\right]$} & {$\left[z_{0}, \cdots, z_{5}\right]$} & $n$ & {$\left[m_{0}, \cdots, m_{9}\right]$} & {$\left[z_{0}, \cdots, z\right.$} \\
\hline 1 & {$[1000000000]$} & [10000] & 26 & [1123456400] & {$[\overline{42} 45 \overline{3}]$} \\
\hline 2 & [1100000000] & [100̄̄o] & 27 & [1123456410] & {$[\overline{42} 45 \overline{2}]$} \\
\hline 3 & [1110000000] & {$[110 \overline{1} 0]$} & 28 & [1123456510] & {$[\overline{43} 45 \overline{2}]$} \\
\hline 4 & [11111000000] & {$[110 \overline{11}]$} & 29 & {$[1123456520]$} & {$[\overline{43} 45 \overline{1}]$} \\
\hline 5 & [1121000000] & [12011] & 30 & [1123456620] & {$[\overline{444} 451]$} \\
\hline 6 & [1122000000] & 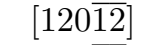 & 31 & [1123456630] & [44450] \\
\hline 7 & [1122100000] & {$[121 \overline{12}]$} & 32 & [1123456730] & {$[\overline{45450]}$} \\
\hline 8 & [1123100000] & {$[121 \overline{13}]$} & 33 & [1123456740] & {$[\overline{45451]}$} \\
\hline 9 & [1123200000] & {$[122 \overline{13}]$} & 34 & [1123456741] & [45351] \\
\hline 10 & [1123210000] & {$[022 \overline{13}]$} & 35 & [1123456751] & {$[\overline{45} 352]$} \\
\hline 11 & [1123310000] & {$[023 \overline{13}]$} & 36 & [1123456752] & {$[\overline{45} 252]$} \\
\hline 12 & [1123320000] & {$[\overline{1} 23 \overline{13}]$} & 37 & [1123456762] & [45253] \\
\hline 13 & [1123420000] & {$[\overline{1} 24 \overline{13}]$} & 38 & [1123456763] & {$[\overline{45} 153]$} \\
\hline 14 & [1123430000] & {$[\overline{2} 24 \overline{13}]$} & 39 & [1123456773] & {$[\overline{45154]}$} \\
\hline 15 & [1123431000] & {$[\overline{2} 240 \overline{3}]$} & 40 & [1123456774] & {$[\overline{45054]}$} \\
\hline 16 & [1123441000] & {$[\overline{3} 240 \overline{3}]$} & 41 & [1123456784] & {$[\overline{45} 055]$} \\
\hline 17 & [1123442000] & {$[\overline{3} 241 \overline{3}]$} & 42 & [1123456785] & [45155] \\
\hline 18 & [1123452000] & {$[\overline{4} 241 \overline{3}]$} & 43 & [2123456785] & [35155] \\
\hline 19 & [1123453000] & {$[\overline{4} 242 \overline{3}]$} & 44 & [2123456786] & {$[\overline{352} 55]$} \\
\hline 20 & [1123453100] & {$[\overline{4} 142 \overline{3}]$} & 45 & [3123456786] & {$[25255]$} \\
\hline 21 & [1123454100] & {$[\overline{4} 143 \overline{3}]$} & 46 & [3123456787] & {$[\overline{25355}$} \\
\hline 22 & [1123454200] & {$[\overline{4} 043 \overline{3}]$} & 47 & [4123456787] & $\overline{3} 55]$ \\
\hline 23 & [1123455200] & {$[\overline{4} 044 \overline{3}]$} & 48 & [4123456788] & $455]$ \\
\hline 24 & [1123455300] & {$[\overline{41} 44 \overline{3}]$} & 49 & [5123456788] & {$[0 \overline{54} 55]$} \\
\hline 25 & [1123456300] & {$[\overline{41} 45 \overline{3}]$} & 50 & [5123456789] & 5.5 \\
\hline
\end{tabular}




\section{Computer code}

\subsection{Mathematica script for the 2D case of the tenfold twin structure $(\kappa=1$ case)}

Finally, we like to give a concise script, written in the language of Mathematica, which shows the implementation of the aforementioned formulas and its corresponding output for 300 points within a single spiral.

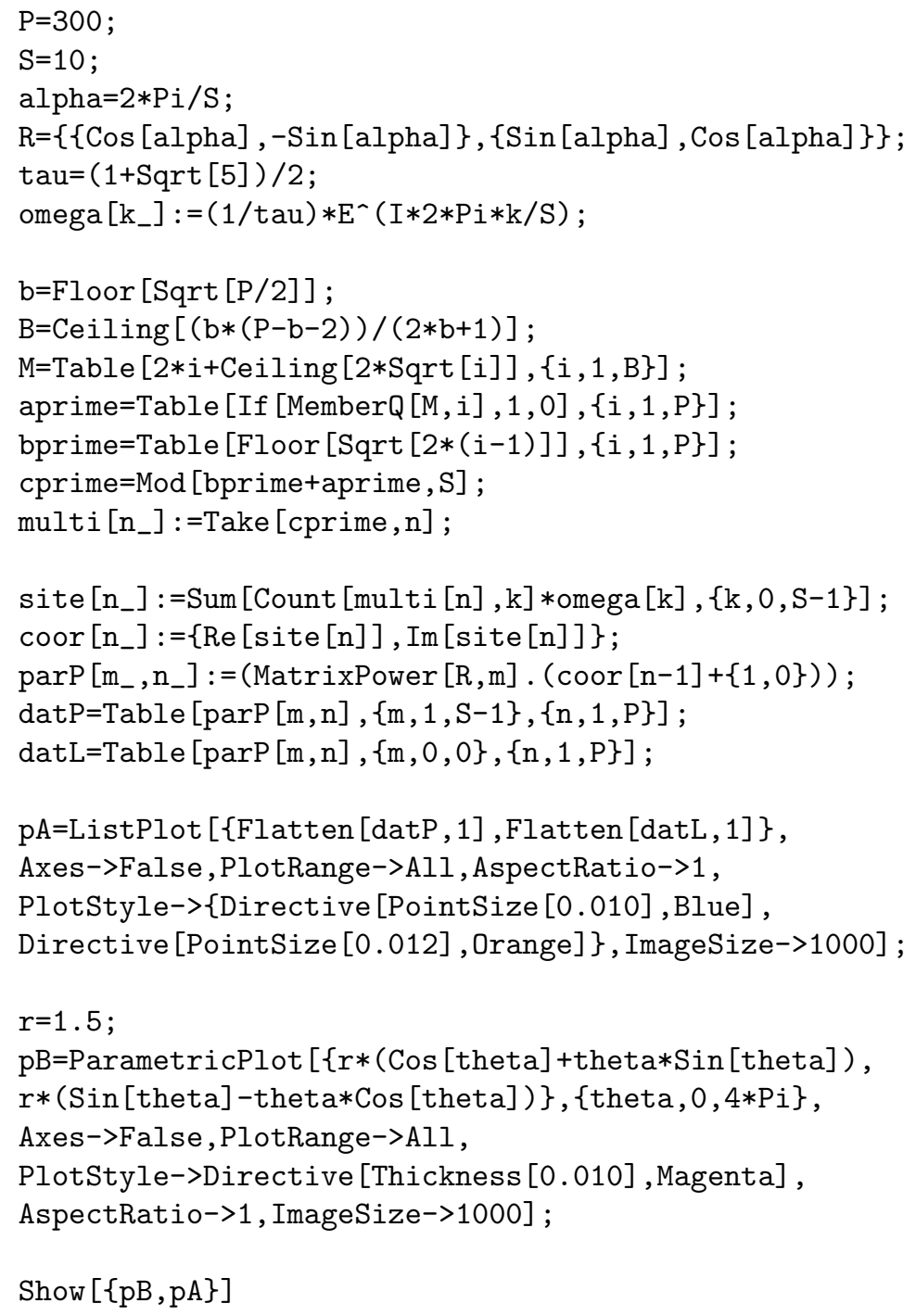

The script consists of five blocks: (i) the declaration of variables - the number of spirals $S$ and the number of points $P$ within a spiral - and the definition of the basis vector function $\omega_{k}$, including the decagonal scaling factor $1 / \tau$; (ii) the generation of the basis vector index sequences $a^{\prime}(n), b^{\prime}(n)$, and $c^{\prime}(n)$; (iii) the parametrization of the points $P(m, n)$ and the generation of the point set; (iv) the generation of the circle involute; and (v) the plotting command. The symbol $B$ denotes the number of 1 's in the string in the interval $[0, P]$. 


\subsection{Mathematica script for the 3D case of the tenfold twin structure ( $\kappa=1$ case)}

In the following we show an Mathematica script for generating a 3D twin structure, scaled according to the experimentally observed lattice parameters of NiZr. Contrary to before we comment each block separately.

First, we define some basic parameters and primitives of the twin pattern, like the number of points $P$ in a spiral, the decagonal symmetry $S$, and the golden ratio scaling factor $\tau$ :

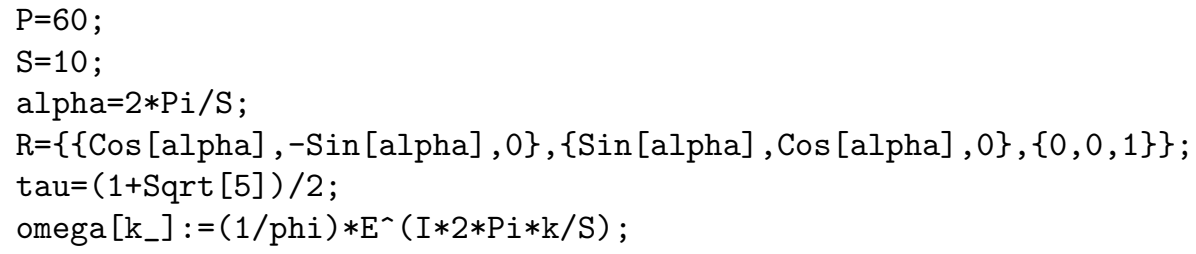

This corresponds to the first block in the $2 \mathrm{D}$ code, except for the $3 \times 3$ rotation matrix. In the second block, the inclination sequence is generated, just as described in the manuscript:

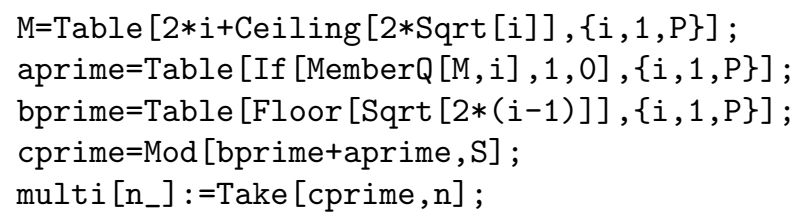

In order to make the $3 \mathrm{D}$ model correspond to the experimentally observed unit cell of CrB type NiZr some scaling factors are introduced:

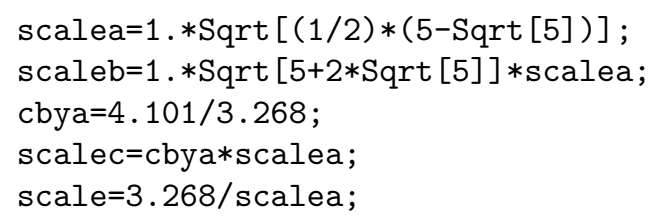

Now, the 3D coordinates are defined, using a matrix multiplication formalism (other ways of implementation exist and might be advantageous in terms of calculation speed when the number of atoms gets comparatively high):

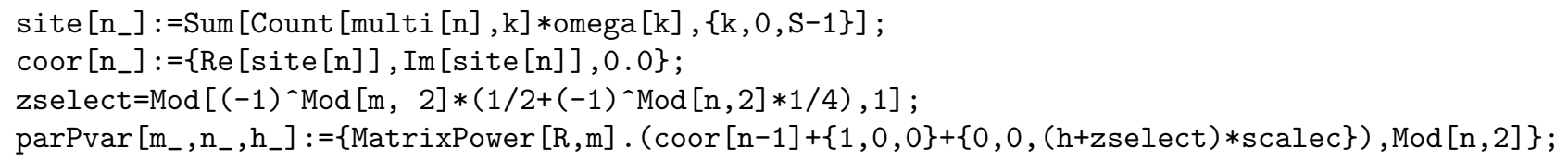

In order to make the point set 'isotropic' a spherical cutoff is introduced:

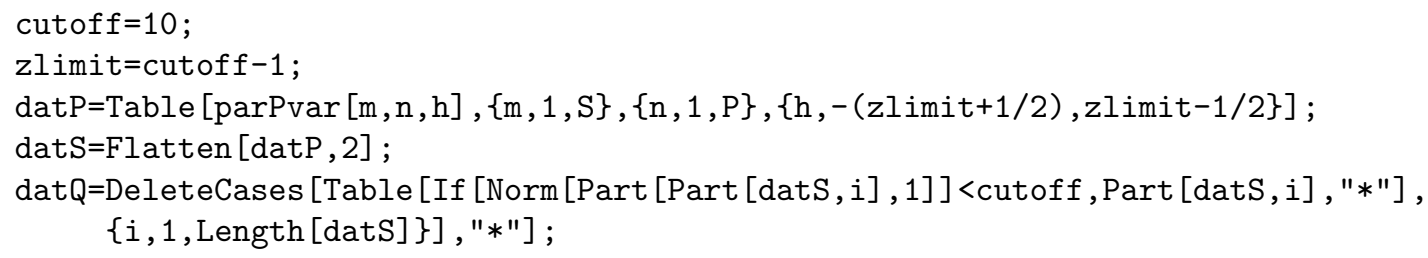

Some parameters are stated for specifying the model's atom size and affecting the position of dummy atoms at the corners of a cube surrounding the model and used for orientation purposes:

radius $=0.12$;

excess $=1.1$; 
The model is created assigning colors according to atom types:

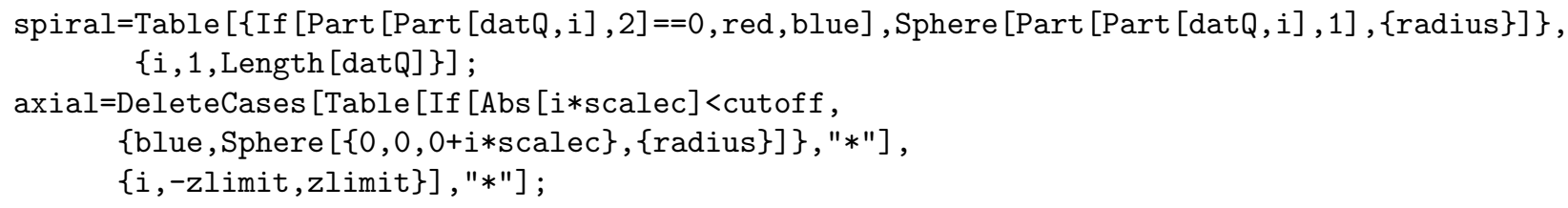

The model is visualized on screen using some additional geometric primitives, like three mutually perpendicular lines forming a basic coordinate system, to give some spatial orientation:

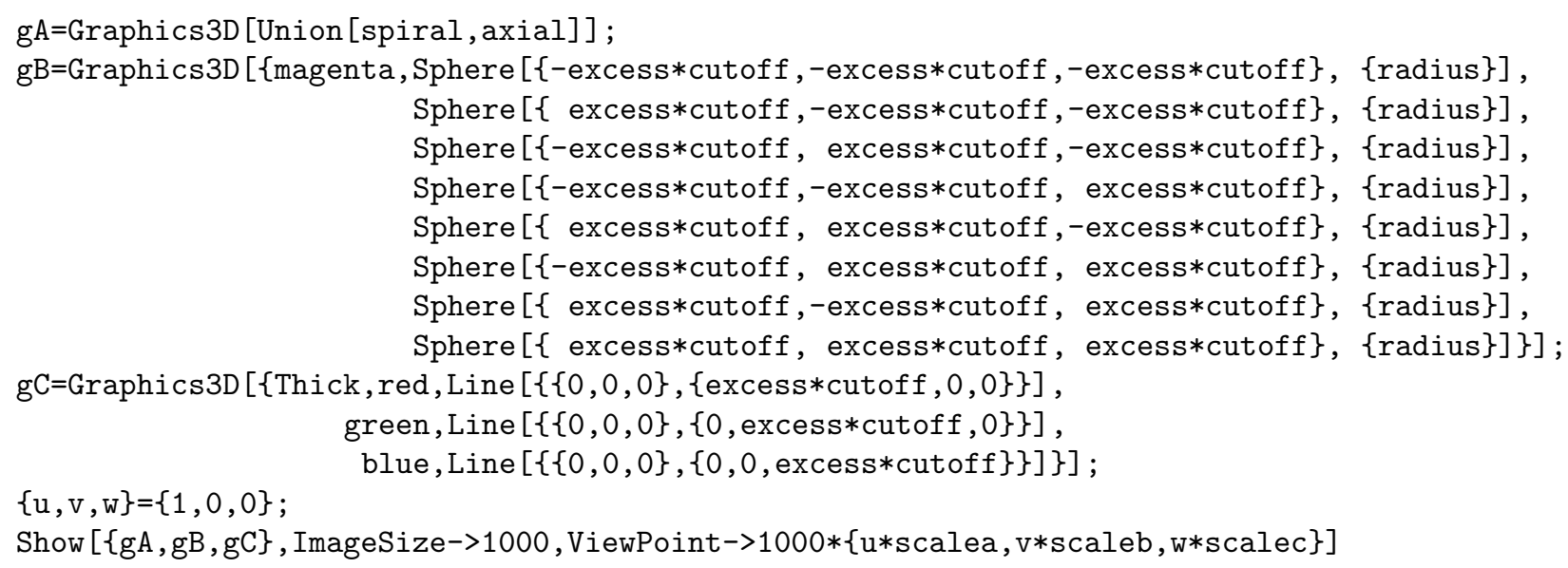

